\title{
STANDING WAVES IN THE FITZHUGH-NAGUMO SYSTEM AND A PROBLEM IN COMBINATORIAL GEOMETRY
}

\author{
MATTHIAS WINTER AND JUNCHENG WEI
}

\begin{abstract}
We show that there is a close relation between standing-wave solutions for the FitzHugh-Nagumo system

$$
\Delta u+u(u-a)(1-u)-\delta v=0, \quad \Delta v-\delta \gamma v+u=0 \text { in } R^{N},
$$$$
u, v \rightarrow 0 \text { as }|x| \rightarrow+\infty
$$

where $0<a<1 / 2$ and $\delta \gamma=\beta^{2} \in(0, a)$, and the following combinatorial problem:

(*) Given $K$ points $Q_{1}, \ldots, Q_{K} \in R^{N}$ with minimum distance 1, find out the maximum number of times that the minimum distance 1 can occur.

More precisely, we show that for any given positive integer $K$, there exists a $\delta_{K}>0$ such that for $0<\delta<\delta_{K}$, there exists a standing-wave solution $\left(u_{\delta}, v_{\delta}\right)$ to the FitzHugh-Nagumo system with the property that $u_{\delta}$ has $K$ spikes $Q_{1}^{\delta}, \ldots, Q_{K}^{\delta}$ and $\left(\frac{1}{l^{\delta}} Q_{1}^{\delta}, \ldots, \frac{1}{l^{\delta}} Q_{K}^{\delta}\right)$ approaches an optimal configuration in $\left(^{*}\right)$, where $l^{\delta}=\min _{i \neq j}\left|Q_{i}^{\delta}-Q_{j}^{\delta}\right|=$ $\frac{1}{\sqrt{a}-\beta} \log \frac{1}{\delta}(1+o(1))$.

Subject class: Primary 35B40, 35B45; Secondary 35J55, 92C15, 92C40
\end{abstract}

Keywords: FitzHugh-Nagumo system, standing waves, optimal configuration, localized energy method.

\section{INTRODUCTION}

In this paper, we study the steady-states for the FitzHugh-Nagumo system in $R^{N}$ [19], [32]. It is a twovariable reaction-diffusion system derived from the Hodgkin-Huxley model for nerve-impulse propagation [26]. In a suitably rescaled fashion it can be written as follows:

$$
(F N) \quad \begin{cases}u_{t}=\Delta u+f(u)-v & \text { in } R^{N}, \\ v_{t}=\Delta v-\delta \gamma v+\delta u & \text { in } R^{N} .\end{cases}
$$

The unknowns $u=u(x, t)$ and $v=v(x, t)$ represent the electric potential and the ion concentration across the cell membrane at a point $x \in R^{N}(N=1,2, \ldots)$ and at a time $t>0$, respectively; $\delta>0$, and $\gamma>0$ are real constants; $\Delta:=\sum_{j=1}^{N} \frac{\partial^{2}}{\partial x_{j}^{2}}$ is the Laplace operator in $R^{N} ; f(u)=u(1-u)(u-a)$ with $a \in(0,1 / 2)$.

In this paper, we consider the steady-state problem of $(\mathrm{FN})$, namely the following elliptic system

$$
\left\{\begin{array}{l}
\Delta u+f(u)-v=0 \quad \text { in } R^{N} \\
\Delta v-\delta \gamma v+\delta u=0 \text { in } R^{N} \\
u(x), v(x) \rightarrow 0 \text { as }|x| \rightarrow+\infty
\end{array}\right.
$$

From now on, we assume that $0<\delta \gamma=\beta^{2}<a=-f^{\prime}(0)$, where $\beta$ is independent of $\delta$. Setting $v=\delta \tilde{v}$ and dropping the tilde we get the system

$$
\left\{\begin{array}{l}
\Delta u+f(u)-\delta v=0 \quad \text { in } R^{N} \\
\Delta v-\beta^{2} v+u=0 \quad \text { in } R^{N} \\
u(x), v(x) \rightarrow 0 \text { as }|x| \rightarrow+\infty
\end{array}\right.
$$

This is the final form of the system which we will study in the rest of the paper. 
The energy functional to (1.2) is given by

$$
E_{\delta}[u]=\frac{1}{2} \int_{R^{N}}|\nabla u|^{2}-\int_{R^{N}} F(u)+\frac{\delta}{2} \int_{R^{N}} u T[u],
$$

where $u \in H^{1}\left(R^{N}\right), F(t)=\int_{0}^{t} f(s) d s$ and $T[u]=\left(-\Delta+\beta^{2}\right)^{-1}[u]$. Namely, (1.2) is the Euler-Lagrange equation to $(1.3)$.

Let $w$ be the unique solution of

$$
\Delta w+f(w)=0, \quad w>0 \quad \text { in } R^{N}, w(0)=\max _{y \in R^{N}} w(y), w(y) \rightarrow 0 \text { as }|y| \rightarrow \infty .
$$

It is well-known that $w$ is radially symmetric: $w(y)=w(|y|)$ and strictly decreasing: $w^{\prime}(r)<0$ for $r>0, r=|y|$. Moreover, we have the following asymptotic behaviour of $w$ :

$$
w(r)=A_{N} r^{-\frac{N-1}{2}} e^{-\sqrt{a} r}\left(1+O\left(\frac{1}{r}\right)\right), \quad w^{\prime}(r)=-A_{N} \sqrt{a} r^{-\frac{N-1}{2}} e^{-\sqrt{a} r}\left(1+O\left(\frac{1}{r}\right)\right),
$$

for $r$ large, where $A_{N}>0$ is a generic constant.

For the uniqueness, we refer to [3], [11] and [36]. Furthermore, $w$ is nondegenerate, i.e.,

$$
\text { Kernel }\left(\Delta+f^{\prime}(w)\right)=\operatorname{span}\left\{\frac{\partial w}{\partial y_{1}}, \ldots, \frac{\partial w}{\partial y_{N}}\right\} \text {. }
$$

We denote the energy of $w$ as

$$
I[w]=\frac{1}{2} \int_{R^{N}}|\nabla w|^{2}-\int_{R^{N}} F(w) .
$$

System (1.1) has been studied among others by DeFigueiredo-Mitidieri [16], Klaasen-Mitidieri [27], KlaasenTroy [28], Lazer-McKenna [30], Reinecke and Sweers ([40], [41], [42], [43]).

Note that our regime $0<\beta^{2}=\gamma \delta<a$ is complementary to [43] and the references thererein and so a different behaviour is expected. Our results show that this is actually the case.

Many of the existence results are analogies of the results for the scalar case $\delta=0$ in [8]. However, numerical results in one- and two-dimensional domains of Sweers and Troy [39] suggest that problem (1.1) admits a rich solution structure. In this regard, the papers [43] and [15] show very interesting behaviour of minimizers of (1.1) which are completely different from the single equation case [8]. The system (1.1) with Neumann boundary conditions has been studied in [34], [35], and [37]. Certain spot-like solutions have been constructed in [38].

In this study, we introduce a new type of spot-like solution, namely a standing wave cluster. More precisely, we rigorously construct a solution of (1.2) in full $N$-dimensional space which for a given positive integer $K$ is concentrated in $K$ spots for $\delta$ small enough. This is new for the FitzHugh-Nagumo system. It shows that the set of solutions of (1.2) has a rich structure.

They are derived by the so-called "localized energy method" based on Liapunov-Schmidt reduction and variational techniques. This poses a restriction on the location of the spots. Namely, we prove the existence of clusters such that the limiting spot locations satisfy the following optimal configuration condition for any dimension $N$ and any number $K$ :

(*) Given $K$ points $Q_{1}, \ldots, Q_{K} \in R^{N}$ with shortest distance 1, find the optimal configuration which maximizes the number of times that the minimum distance 1 can occur. 
We denote the optimal number in $\left(^{*}\right)$ by $m(N, K)$. The optimal configurations for $N=2, K=4,6,7$ are depicted as follows:

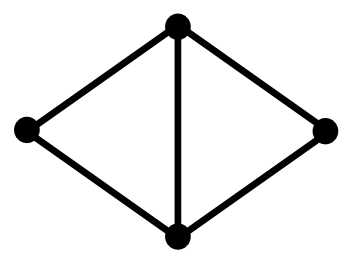

$K=4$

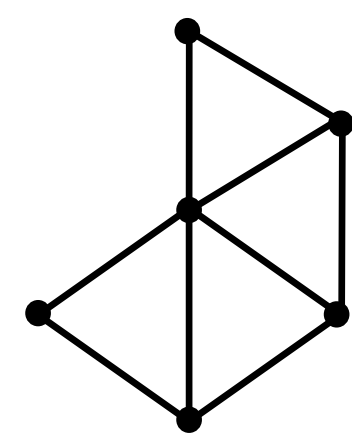

$K=6$

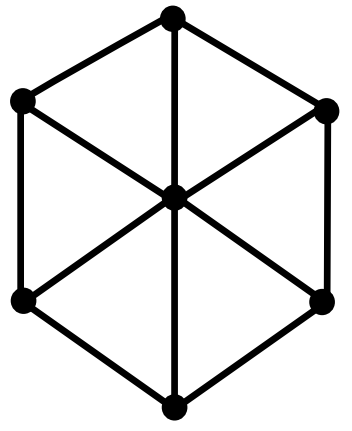

$K=7$

\section{Figure 1: Optimal Configurations for $\mathrm{K}=4,6,7$}

Thus the location of spots is related to the optimal configuration problem in combinatorial geometry. We will discuss this problem in Section 2.

The following is the main result of this paper.

Theorem 1.1. For any given integer $K \geq 1$ there exists a $\delta_{0}>0$ such that for all delta with $0<\delta<\delta_{0}$ problem (1.2) admits a solution $\left(u_{\delta}, v_{\delta}\right)$ with the following properties

(1) $u_{\delta}(y)=\sum_{i=1}^{K}\left(w\left(y-Q_{i}^{\delta}\right)+o(1)\right)$ uniformly for $y \in R^{N}$, where $w$ is defined by (1.4).

(2) For exactly $m(N, K)$ pairs $i \neq j$ we have

$$
\left|Q_{i}^{\delta}-Q_{j}^{\delta}\right|=\frac{1}{\sqrt{a}-\beta} \log \frac{1}{\delta}(1+o(1)) .
$$

For all other $i \neq j$, there exists some $\mu>0$ such that

$$
\left|Q_{i}^{\delta}-Q_{j}^{\delta}\right| \geq\left(\frac{1}{\sqrt{a}-\beta}+\mu\right) \log \frac{1}{\delta}
$$

Furthermore, $\frac{1}{l^{\delta}}\left(Q_{1}^{\delta}, \ldots, Q_{K}^{\delta}\right)$ approaches an optimal configuration in $\left(^{*}\right)$, where $l^{\delta}=\min _{i \neq j}\left|Q_{i}^{\delta}-Q_{j}^{\delta}\right|$.

Remark: The proof of Theorem 1.1 uses a sequence of lemmata. We assume that $\delta_{0}$ is chosen so small that all of these are true.

Using symmetry, we can also construct other types of solutions. Now the spots are located near the vertices of a regular polygon or two concentric polygons in a two-dimensional plane.

Theorem 1.2. For all $\delta$ with $0<\delta<\delta_{0}$ problem (1.2) also has a solution $\left(u_{\delta}, v_{\delta}\right)$ with property (1) of Theorem 1.1. Further, for this solution the following cases are all possible: (i) $Q_{i}^{\delta}$ are the vertices of a regular $K$-polygon 
in a two-dimensional plane; (ii) if $K \geq 6$ and $K$ is even, $Q_{i}^{\delta}$ are the vertices of two concentric regular polygons in a two-dimensional plane.

Remarks: 1. By our method, it is also possible to construct solutions concentrating on multiple rings of regular polygons.

2. We do not know if our solutions are positive. While the function $w>0$ defined in (1.4) is positive, we expect that the function $\hat{w}$ which will be defined in (3.5) is negative.

Let us now summarize the proof of Theorem 1.1.

We define a configuration space

$$
\Gamma=\left\{\left(Q_{1}, \ldots, Q_{K}\right) \in R^{N K}\left|\sum_{j=1}^{K} Q_{j}=0, \quad(1-\mu) \frac{1}{\sqrt{a}-\beta} \log \frac{1}{\delta} \leq\right| Q_{i}-Q_{j} \mid \leq\left(\log \frac{1}{\delta}\right)^{2}\right\},
$$

where we choose $\mu$ such that

$$
0<\mu<\min \left\{\frac{\beta}{100}, \frac{1}{10}\left(1-\frac{\beta}{\sqrt{a}}\right)\right\} .
$$

Since $0<\beta^{2}<a<1 / 2$, this implies that $\mu$ is very small (compared to the other parameters in the problem and absolutely). Also $\mu$ becomes smaller and smaller the closer $\beta$ approximates $\sqrt{a}$.

Note that we may introduce the condition $\sum_{j=1}^{K} Q_{j}=0$ by the translational invariance. The reason for introducing it is to have a compact set $\bar{\Gamma}$.

Let $\mathbf{Q}=\left(Q_{1}, \ldots, Q_{K}\right) \in \bar{\Gamma}$.

Theorem 1.1 is proved by the so-called "localized energy method", a combination of the Liapunov-Schmidt reduction method and the variational principle. The Liapunov-Schmidt reduction method has been introduced and used in a lot of papers. See [1], [2], [3], [4], [5], [20], [21], [22], [29], [44], [45] and the references therein. A combination of the Liapunov-Schmidt reduction method and the variational principle was used in [3], [13], [14], [21], [22] and [23]. We shall follow the procedure in [21]. This enables us to reduce the energy functional $E_{\delta}$ to finite dimensions. Then local maxima for the reduced energy are found by maximizing $E_{\delta}$ over $\bar{\Gamma}$ and showing that this maximum actually belongs to the interior of $\Gamma$.

Theorem 1.2 is proved by the same approach and by using symmetry reduction.

We describe some related work on clusters. Clusters which are located at the boundary of a bounded domain have been constructed in [13], [23]. However, the configuration of the cluster is unknown. Multiple clusters in an interval have been derived in [46] for the Gierer-Meinhardt system. For clusters for the Gierer-Meinhardt system in $R^{1}$, see [7] and [12]. For clustered spots located on regular polygons for Gierer-Meinhardt system in $R^{2}$ see [17]. We are unaware of any previous results on clusters in the whole $R^{N}, N \geq 3$. This paper seems to be the first.

Let us now give an outline of the paper. In Section 2 we study the geometrical problem $\left(^{*}\right)$. In Section 3 we derive the key energy estimates. In Section 4 we reduce the problem to finite dimensions by the Liapunov-Schmidt reduction method. In Section 5, we compute the reduced energy and show that a critical point for the reduced energy gives rise to a solution to (1.2). In Section 6 we solve the reduced problem by energy maximization in the set $\bar{\Gamma}$ defined in (1.8) and derive Theorem 1.1. Finally, in Section 7 we use the same approach plus symmetry reduction to prove Theorem 1.2 . 
Throughout this paper, the constants $c_{1}, c_{2}, \ldots$ are generic constants depending on $N, a$ and $\beta$ only.

We write

$$
f(u)=-a u+(a+1) u^{2}-u^{3}=-a u+g(u), \text { where } g(u)=(a+1) u^{2}-u^{3} .
$$

Let $G[u]=\int_{0}^{u} g(s) d s$. We also define

$$
\epsilon:=\delta^{\sqrt{a} /(\sqrt{a}-\beta)} .
$$

Acknowledgments. The research of JW is supported by an Earmarked Grant from RGC of Hong Kong. MW thanks the Department of Mathematics at CUHK for their kind hospitality. JW and MW thank Professor Thomas Au and Professor K.M. Yeung for useful discussions.

\section{Optimal Configurations For Problem $(*)$}

Since problem $(*)$ plays an important role in the formation of cluster, we study the properties of $(*)$ in this section.

To begin with, let us fix $K$ points $\left(Q_{1}, \ldots, Q_{K}\right) \in R^{N K}$ and define

$$
R\left[Q_{1}, \ldots, Q_{K}\right]=\text { the number of times that the minimum distance is attained. }
$$

Set

$$
\Sigma:=\left\{\left(Q_{1}, \ldots, Q_{K}\right) \in R^{N K}\left|\sum_{j=1}^{K} Q_{j}=0, \min _{i \neq j}\right| Q_{i}-Q_{j} \mid=1\right\} .
$$

Then Problem $(*)$ can be restated as the following maximization problem

$$
m(N, K):=\sup _{\left(Q_{1}, \ldots, Q_{K}\right) \in \Sigma} R\left[Q_{1}, \ldots, Q_{K}\right] .
$$

The problem is to determine this number $m(N, K)$ and also characterize the configurations for which such an optimal number is achieved.

We state the following simple lemma.

Lemma 2.1. In problem (2.3) the maximum is always attained by some optimal configuration. The graph of shortest distances is connected and contains all points $Q_{j}$. Moreover,

$$
m(N, K)>\sum_{k_{1}+\ldots+k_{l}=K, k_{i}>0} m\left(N, k_{i}\right)
$$

and

$$
\left|Q_{i}-Q_{j}\right| \leq K
$$

\section{Proof:}

Let $\left(Q_{1}, \ldots, Q_{K}\right) \in \Sigma$. Without loss of generality, we may assume that $\left|Q_{1}-Q_{2}\right|=1$. We will show that there exists an optimal configuration lying in $\Sigma$.

In fact, if there exists a subset whose points have distance strictly greater than 1 from another subset, we can bring these two subsets so close to each other that there are points $Q_{i} \neq Q_{j}$ on their respective convex hulls which have unit distance. In this way $R\left[Q_{1}^{n}, \ldots, Q_{K}^{n}\right]$ increases. This implies (2.4). Since there are only finitely 
many subsets, after finitely many optimization steps, the graph of minimal distances is connected and each point $Q_{i}$ is a vertex of it. This implies (2.5).

So, we consider those configurations only whose graph of minimal distances is connected and contains each point $Q_{i}$ as a vertex. Because of $R\left[Q_{1}, \ldots, Q_{K}\right] \leq K(K-1) / 2$ the function $R\left[Q_{1}, \ldots, Q_{K}\right]$ is bounded from above. Since it only has finitely many values, the supremum is attained and there exists an optimal configuration lying in $\Sigma$.

In general, it is difficult to find the number $m(N, K)$ and this problem has a long history. See [6] and [18] for surveys on this subject. That $m(2, K)<3 K$ follows from the observation that six equal circular discs may be placed around another disc of the same size so that the central one is touched by all others and that neighboring discs touch each other. Erdös showed that

$$
3 K-c_{1} K^{1 / 2}<m(2, K)<3 K-c_{2} K^{1 / 3}
$$

and conjectured that

$$
m\left(2,3 K^{2}+3 K+1\right)=9 K^{2}+6 K
$$

by considering a large array of a hexagonal packing of circles. Then Harborth [25] proved this conjecture and further showed the following striking result

$$
m(2, K)=\left[3 K-(12 K-3)^{1 / 2}\right] \quad \text { for all } K,
$$

where $[x]=n$ is the largest integer $n$ with $n \leq x$.

In an analogous three-dimensional situation it is possible to place 12 balls of equal size around another one of the same size, all touching the first but not overlapping with it or with each other. The question if it is possible to place a 13th ball was the subject of a long-standing argument between Sir Isaac Newton and David Gregory. Newton believed 12 to be correct, but this was not proved until 180 years later. It was R. Hoppe who eventually proved that Newton had been right (see Bender [6]). There have been several simpler proofs since then, for example [31].

Hoppe's results imply that $m(3, K)<6 K$, and in fact for $K$ large

$$
m(3, K)=6 K+O\left(K^{2 / 3}\right)
$$

because of effects coming from the surface. For $N>3$ results on the maximum number of exterior spheres touching a central one are known only in a few cases, for example it is 240 for $N=8$ and 196560 for $N=24$. For further information, we refer to [9], [10], [24].

In some cases, however, the answer is easy to determine. For example, $m(2,3)=3$ corresponds to the regular triangle. Further, $m(2,4)=5$ and this is achieved by putting two regular triangles together such that they have one common side. The case $m(2,7)=12$ corresponds to a hexagon with a center. See Figure 1 . The result $m(3,4)=6$ corresponds to the regular tetrahedron. Note that in general $m(N, K) \leq K(K-1) / 2$.

We remark that condition $(*)$ is forced on us since we want to find cluster which maximize the energy. It therefore is not merely a technical condition but characterizes certain multi-spot solutions with the property that it is not possible for one spot (or several spots) to move away from the rest. 


\section{Key Energy Estimate}

Let $w$ be the ground state solution of (1.4).

Let $K_{\beta}$ be the fundamental solution of $\Delta K_{\beta}-\beta^{2} K_{\beta}+\delta_{0}=0$ in $R^{N} \backslash\{0\}$, where $\delta_{0}$ is the Dirac delta distribution at zero. It is easy to see that

$$
K_{\beta}(|y|)=C_{N}|y|^{-\frac{N-1}{2}}\left(1+O\left(\frac{1}{|y|}\right)\right) e^{-\beta|y|} \text { for }|y|>1 .
$$

Let $\Psi(y)$ be the unique solution of

$$
\Delta \Psi-\beta^{2} \Psi+w(y)=0 \text { in } R^{N}
$$

Recall that by the previous equation we can define $\Psi=T[w]$. Since $\beta<\sqrt{a}$, we have for $|y|>1$,

$$
\Psi(y)=B_{N}\left(1+O\left(\frac{1}{|y|}\right)\right) K_{\beta}(|y|),
$$

for some $B_{N}>0$.

We begin with the following theorem, which is the special case $K=1$ of Theorem 1.1.

Theorem 3.1. For $0<\delta<\delta_{0}$ the problem

$$
\left\{\begin{array}{l}
\Delta u-a u+g(u)-\delta v=0, \quad \Delta v-\beta^{2} v+u=0 \text { in } R^{N}, \\
u=u(|y|), v=v(|y|), \quad u, v \rightarrow 0 \text { as }|y| \rightarrow+\infty
\end{array}\right.
$$

has a unique (radially symmetric) solution $\left(u_{\delta}, v_{\delta}\right)$, where $u_{\delta}=w_{\delta}(|y|)$ such that

$$
w_{\delta}(|y|)=w(|y|)+\delta \hat{w}(|y|)+O\left(\delta^{3 / 2} e^{-\mu|y|}\right),
$$

and $\hat{w}(|y|)$ is the unique radially symmetric solution to the following problem

$$
\Delta \hat{w}-a \hat{w}+g^{\prime}(w) \hat{w}-\Psi=0 \quad \text { in } R^{N}, \hat{w} \in H^{1}\left(R^{N}\right)
$$

and $\Psi$ is defined in (3.2).

Note: The second equatino in problem (3.4) can be rewritten as $v_{\delta}=T\left[u_{\delta}\right]$, using the notation introduced after (3.2). Therefore problem (3.4) can be rephrased as a nonlocal equation for $u$. In other words, knowing $u$ means that the system has been solved. That is why it is only necessary to give an expansion for $u_{\delta}($ see $(3.5))$.

Proof: Let us denote

$$
L_{0}[\phi]:=\Delta \phi-a \phi+g^{\prime}(w) \phi
$$

For $p>N$, we define

$$
W_{\mu, r}^{2, p}\left(R^{N}\right)=\left\{u=u(|y|) \mid e^{\mu|y|} u(|y|) \in W^{2, p}\left(R^{N}\right)\right\} .
$$

Since $L_{0}$ is nondegenerate, it is easy to see that the operator $L_{0}$ is an invertible operator from $W_{\mu, r}^{2, p}\left(R^{N}\right)$ to $L_{\mu, r}^{p}\left(R^{N}\right)$. We write (3.4) in operator form:

$$
S_{\delta}[u]:=\Delta u-a u+g(u)-\delta T[u]=0
$$

Note that by our construction

$$
\left|S_{\delta}[w+\delta \hat{w}]\right|=\left|g(w+\delta \hat{w})-g(w)-\delta g^{\prime}(w) \hat{w}-\delta^{2} T[\hat{w}]\right| \leq C \delta^{2} e^{-\mu|y|} .
$$


We put

$$
u=w(r)+\delta \hat{w}(r)+\delta^{1+\gamma} \phi,
$$

where $0<\gamma<1$. Without loss of generality, we choose $\mu=1 / 2$. Then we see that

$$
\delta^{-3 / 2} S_{\delta}[u]=\Delta \phi-a \phi+g^{\prime}(w+\delta \hat{w}) \phi-\delta T[\phi]+N[\phi]+O\left(\delta^{1 / 2} e^{-\mu|y|}\right),
$$

where $|N[\phi]| \leq C \phi^{2}$ is a higher order term. Because of the invertibility of the operator $L_{0}$ a standard perturbation argument shows that the new linear operator $\Delta \phi-a \phi+g^{\prime}(w+\delta \hat{w}) \phi-\delta T[\phi]$ is also an invertible operator from $W_{\mu, r}^{2, p}$ to $L_{\mu, r}^{p}$. Now the result follows from the contraction mapping theorem. (The details are as in the proof of Lemma 4.3 below, where the more difficult situation of a non-invertible operator is considered. Therefore we skip them here).

Let $w_{\delta}(|y|)$ be the unique solution to (3.4).

Let $\left(Q_{1}, \ldots, Q_{K}\right) \in \Gamma$. We define

$$
w_{i}=w\left(y-Q_{i}\right), \Psi_{i}=\Psi\left(y-Q_{i}\right), w_{\delta, i}(y)=w_{\delta}\left(y-Q_{i}\right), i=1, \ldots, K, w_{\delta, \mathbf{Q}}=\sum_{i=1}^{K} w_{\delta, i} .
$$

Note that for $\mathbf{Q}:=\left(Q_{1}, \ldots, Q_{K}\right) \in \Gamma$, we have

$$
w\left(\left|Q_{i}-Q_{j}\right|\right) \leq C e^{-(1-\mu) \frac{\sqrt{a}}{\sqrt{a}-\beta} \log \frac{1}{\delta}} \leq C \epsilon^{1-\mu}
$$

where $\epsilon$ is defined at (1.10). Similarly,

$$
\delta\left|\Psi\left(\left|Q_{i}-Q_{j}\right|\right)\right| \leq C \delta e^{-(1-\mu) \frac{\beta}{\sqrt{a}-\beta} \log \frac{1}{\delta}} \leq C \epsilon^{1-\mu}
$$

since $\beta<\sqrt{a}$.

The following useful lemma is an easy consequence of Lebesgue's Dominated Convergence Theorem.

Lemma 3.2. Let $f_{1}(|y|)$ and $f_{2}(|y|)$ be two $C^{2}$ functions such that for $|y|$ large

$$
f_{1}(|y|) \sim C_{1}|y|^{-m_{1}} e^{-\gamma_{1}|y|}, f_{2}(|y|) \sim C_{2}|y|^{-m_{2}} e^{-\gamma_{2}|y|}
$$

where $\gamma_{1} \geq \gamma_{2}>0, C_{1}>0, C_{2}>0$, and $m_{1}, m_{2}$ are arbitrary constants. Then we have

$$
C e^{-\gamma_{2}(1+\mu)\left|Q_{1}-Q_{2}\right|} \leq \int_{R^{N}} f_{1}\left(y-Q_{1}\right) f_{2}\left(y-Q_{2}\right) \leq C e^{-\gamma_{2}(1-\mu)\left|Q_{1}-Q_{2}\right|}
$$

and when $\gamma_{1}>\gamma_{2}>0$,

$$
\frac{1}{f_{2}\left(\left|Q_{1}-Q_{2}\right|\right)} \int_{R^{N}} f_{1}\left(y-Q_{1}\right) f_{2}\left(y-Q_{2}\right) d x \rightarrow \gamma_{0}>0 \quad \text { as }\left|Q_{1}-Q_{2}\right| \rightarrow \infty,
$$

where

$$
\gamma_{0}=\int_{R^{N}} f_{1}(y) e^{-\gamma_{2} y_{1}} d y
$$

Moreover, when $\gamma_{1}>\gamma_{2}>0$, the function

$$
\int_{R^{N}} f_{1}\left(y-Q_{1}\right) f_{2}\left(y-Q_{2}\right) d y
$$

is a $C^{2}$ function in $\left|Q_{1}-Q_{2}\right|$ and (3.13) holds in $C^{2}$ sense. 
We also define two functions

$$
\begin{gathered}
\alpha\left(\left|Q_{i}-Q_{j}\right|\right)=\int_{R^{N}} g\left(w\left(y-Q_{i}\right)\right) w\left(y-Q_{j}\right)=\int_{R^{N}} g(w(y)) w\left(y+Q_{i}-Q_{j}\right) d y, \\
\gamma\left(\left|Q_{i}-Q_{j}\right|\right)=\int_{R^{N}} w\left(y-Q_{i}\right) \Psi\left(y-Q_{j}\right)=\int_{R^{N}} w(y) \Psi\left(y+Q_{i}-Q_{j}\right) d y .
\end{gathered}
$$

Note that for $\mathbf{Q}=\left(Q_{1}, \ldots, Q_{K}\right) \in \Gamma$ Lemma 3.2 gives

$$
\alpha\left(\left|Q_{i}-Q_{j}\right|\right) \sim w\left(\left|Q_{i}-Q_{j}\right|\right) \leq C \epsilon^{1-\mu}
$$

using (3.9) and

$$
\delta \gamma\left(\left|Q_{i}-Q_{j}\right|\right) \sim \delta \Psi\left(\left|Q_{i}-Q_{j}\right|\right) \leq C \epsilon^{1-\mu} .
$$

using (3.10).

With the help of Lemma 3.2, we derive the following key energy estimate:

Lemma 3.3. For any $\mathbf{Q}=\left(Q_{1}, \ldots, Q_{K}\right) \in \bar{\Gamma}$ and $0<\delta<\delta_{0}$ we have

$$
\begin{gathered}
E_{\delta}\left[w_{\delta, \mathbf{Q}}\right]=K E_{\delta}\left[w_{\delta}\right]-\sum_{i<j} \alpha\left(\left|Q_{i}-Q_{j}\right|\right) \\
+c_{1} \delta \sum_{i<j} \gamma\left(\left|Q_{i}-Q_{j}\right|\right)+O\left(\epsilon^{1+4 \mu}\right),
\end{gathered}
$$

where $c_{1}$ is a real positive constant, $\mu$ is given by (1.9) and the functions $\alpha$ and $\gamma$ have been defined in (3.15) and (3.16), respectively.

Proof. We compute

$$
\begin{gathered}
E_{\delta}\left[w_{\delta, \mathbf{Q}}\right]=\frac{1}{2} \int_{R^{N}}\left|\nabla w_{\delta, \mathbf{Q}}\right|^{2}-\int_{R^{N}} F\left(w_{\delta, \mathbf{Q}}\right) \\
+\frac{\delta}{2} \int_{R^{N}}\left(\sum_{j=1}^{K} w_{\delta}\left(y-Q_{j}\right)\right)\left(\sum_{i=1}^{K} T\left[w_{\delta}\left(y-Q_{i}\right)\right]\right) .
\end{gathered}
$$

Using (3.4), we can decompose $E_{\delta}$ into two parts:

$$
E_{\delta}\left[w_{\delta, \mathbf{Q}}\right]=: I_{1}+I_{2},
$$

where

and

$$
I_{1}=\sum_{i=1}^{K} E_{\delta}\left[w_{\delta, i}\right]=K E_{\delta}\left[w_{\delta}\right]
$$

Note that

$$
I_{2}=-\int_{R^{N}}\left[G\left(\sum_{i=1}^{K} w_{\delta, i}\right)-\sum_{i=1}^{K} G\left(w_{\delta, i}\right)-\sum_{i<j} g\left(w_{\delta, i}\right) w_{\delta, j}\right] .
$$

$$
I_{2}=-\int_{R^{N}}\left[G\left(\sum_{i=1}^{K} w_{\delta, i}\right)-\sum_{i=1}^{K} G\left(w_{\delta, i}\right)-\sum_{i \neq j} g\left(w_{\delta, i}\right) w_{\delta, j}\right]-\int_{R^{N}} \sum_{i<j} g\left(w_{\delta, i}\right) w_{\delta, j} .
$$

The first term in $I_{2}$ can be estimated as

$$
\int_{R^{N}}\left|G\left(\sum_{i=1}^{K} w_{\delta, i}\right)-\sum_{i=1}^{K} G\left(w_{\delta, i}\right)-\sum_{i \neq j} g\left(w_{\delta, i}\right) w_{\delta, j}\right|
$$




$$
\begin{gathered}
\leq C \sum_{i \neq j} \int_{R^{N}}\left|w_{\delta, i}\right|^{2}\left|w_{\delta, j}\right|^{2} \\
\leq C \sum_{i \neq j} \int_{R^{N}}\left|w_{i}+\delta \hat{w}_{i}\right|^{2}\left|w_{j}+\delta \hat{w}_{j}\right|^{2} \leq C \epsilon^{1+4 \mu} .
\end{gathered}
$$

For the second term in $I_{2}$, we have

$$
\begin{aligned}
\int_{R^{N}} g\left(w_{\delta, i}\right) w_{\delta, j} & =\int_{R^{N}}\left[g\left(w_{i}\right)+\delta g^{\prime}\left(w_{i}\right) \hat{w}_{i}\right]\left[w_{j}+\delta \hat{w}_{j}\right]+O\left(\delta^{2} \sum_{i \neq j} \gamma\left(\left|Q_{i}-Q_{j}\right|\right)\right) \\
& =\alpha\left(\left|Q_{i}-Q_{j}\right|\right)+\delta \int_{R^{N}} g\left(w_{i}\right) \hat{w}_{j}+O\left(\epsilon^{1+4 \mu}\right) .
\end{aligned}
$$

Note that

$$
\begin{gathered}
\delta \int_{R^{N}} g\left(w_{i}\right) \hat{w}_{j}=-\delta \int_{R^{N}}\left[\Delta w_{i}-a w_{i}\right] \hat{w}_{j} \\
=-\delta \int_{R^{N}}\left[\Delta \hat{w}_{j}-a \hat{w}_{j}\right] w_{i} \\
=-\delta \int_{R^{N}} \Psi_{j} w_{i}+\delta \int_{R^{N}} g^{\prime}\left(w_{j}\right) \hat{w}_{j} w_{i} \text { by }(3.6) \\
=-\delta \gamma\left(\left|Q_{i}-Q_{j}\right|\right)+O\left(\epsilon^{1+4 \mu}\right) \text { by }(3.16)
\end{gathered}
$$

we get

$$
I_{2}=\alpha\left(\left|Q_{i}-Q_{j}\right|\right)-\delta \gamma\left(\left|Q_{i}-Q_{j}\right|\right)+O\left(\epsilon^{1+4 \mu}\right) .
$$

Summarizing the results for $I_{1}$ and $I_{2}$, the proof is finished.

We are also in need of the following lemma on error estimates.

Lemma 3.4. Suppose $\left(Q_{1}, \ldots, Q_{K}\right) \in \Gamma$ and $0<\delta<\delta_{0}$. Then we have

$$
\left|\Delta w_{\delta, \mathbf{Q}}-a w_{\delta, \mathbf{Q}}+g\left(w_{\delta, \mathbf{Q}}\right)-\delta T\left[w_{\delta, \mathbf{Q}}\right]\right| \leq C \epsilon^{1-2 \mu} e^{-\mu \min _{i=1, \ldots, K}\left|y-Q_{i}\right|}
$$

Proof: Note

$$
\Delta w_{\delta, \mathbf{Q}}-a w_{\delta, \mathbf{Q}}+g\left(w_{\delta, \mathbf{Q}}\right)-\delta T\left[w_{\delta, \mathbf{Q}}\right]=g\left(w_{\delta, \mathbf{Q}}\right)-\sum_{j=1}^{K} g\left(w_{\delta, j}\right) .
$$

We divide the domain into $K+1$ parts: $\Omega_{j}=\left\{y|| y-Q_{j}\left|\leq \frac{1}{2} \min _{i \neq j}\right| Q_{i}-Q_{j} \mid\right\}, j=1, \ldots, K, \Omega_{K+1}=$ $R^{N} \backslash \cup_{j=1}^{K} \Omega_{j}$. On each $\Omega_{j}, j=1, \ldots, K$ we have $\left|w_{\delta, j}\right| \geq\left|w_{\delta, i}\right|$ for all $i \neq j$. We have on $\Omega_{j}$

$$
\left|g\left(w_{\delta, j}+\sum_{i \neq j} w_{\delta, i}\right)-g\left(w_{\delta, j}\right)-\sum_{i \neq j} g\left(w_{\delta, i}\right)\right| \leq C \sum_{i \neq j}\left|g^{\prime}\left(w_{\delta, j}\right) w_{\delta, i}\right| \leq C \epsilon^{1-2 \mu} e^{-\mu\left|y-Q_{j}\right|} .
$$

On $\Omega_{K+1}$, we have

$$
\left|w_{\epsilon, j}\right| \leq C e^{-\frac{(1-\mu)}{2} \frac{\sqrt{a}}{\sqrt{a}-\beta} \log \frac{1}{\delta}} e^{-\mu / 2 \min _{i=1, \ldots, K}\left|y-Q_{i}\right|} \leq C \epsilon^{(1-\mu) / 2} e^{-\mu / 2 \min _{i=1, \ldots, K}\left|y-Q_{i}\right|} .
$$

So, on $\Omega_{K+1}$,

$$
\left|g\left(w_{\delta, \mathbf{Q}}\right)-\sum_{j=1}^{K} g\left(w_{\delta, j}\right)\right| \leq C \sum_{j=1}^{K}\left|w_{\epsilon, j}\right|^{2} \leq C \epsilon^{1-2 \mu} e^{-\mu \min _{i=1, \ldots, K}\left|y-Q_{i}\right|}
$$




\section{Liapunov-Schmidt Reduction}

Let

$$
S_{\delta}[u]:=\Delta u-a u+g(u)-\delta T[u] .
$$

We now introduce the functional-analytic framework. For $u, v \in H^{1}\left(R^{N}\right)$, we define the following scalar product:

$$
(u, v)=\int_{R^{N}}[\nabla u \nabla v+a u v] d y .
$$

Then, using the relation

$$
\Delta \frac{\partial w_{i}}{\partial Q_{i, j}}-a \frac{\partial w_{i}}{\partial Q_{i, j}}+g^{\prime}\left(w_{i}\right) \frac{\partial w_{i}}{\partial Q_{i, j}}=0
$$

we get that the orthogonality to the function $\frac{\partial w_{i}}{\partial Q_{i, j}}$ in $H^{1}\left(R^{N}\right)$ is equivalent to orthogonality to the function

$$
Z_{i, j}:=g^{\prime}\left(w_{i}\right) \frac{\partial w_{i}}{\partial Q_{i, j}}
$$

in $L^{2}\left(R^{N}\right)$ with the usual scalar product

$$
<u, v>=\int_{R^{N}} u v d y .
$$

This section is devoted to the study of the following system in $(\phi, \beta)$ :

$$
S_{\delta}\left[w_{\delta, \mathbf{Q}}+\phi\right]=\sum_{i, j} \beta_{i j} Z_{i, j}, \quad<\phi, Z_{i, j}>=0, \quad i=1, \ldots, K, j=1, \ldots, N .
$$

To this end, we introduce the following weighted norm for a function defined on $R^{N}$ : Let $0<\mu<1$ be given in (1.9). For $\left(Q_{1}, \ldots, Q_{K}\right) \in \bar{\Gamma}$ we define

$$
\|\phi\|_{*}:=\sup _{y \in R^{N}} e^{\mu \min _{i=1, \ldots, K}\left|y-Q_{i}\right|}|\phi(y)| .
$$

Introduce the spaces

$$
H_{*}^{2}\left(R^{N}\right)=\left\{u \in H^{2}\left(R^{N}\right) \mid\|u\|_{*}<\infty\right\}
$$

and

$$
L_{*}^{\infty}\left(R^{N}\right)=\left\{u \in L^{\infty}\left(R^{N}\right) \mid\|u\|_{*}<\infty\right\} .
$$

We first consider a linear problem: $h \in L_{*}^{\infty}\left(R^{N}\right)$ being given, find a function $\phi$ satisfying

$$
\left\{\begin{array}{l}
L_{\delta}[\phi]:=\Delta \phi-a \phi+g^{\prime}\left(w_{\delta, \mathbf{Q}}\right) \phi-\delta T[\phi]=h+\sum_{i, j} \beta_{i j} Z_{i, j} \\
\phi \in H_{*}^{2}\left(R^{N}\right), \quad<\phi, Z_{i, j}>=0
\end{array}\right.
$$

for some constants $\beta_{i, j} \in R$.

The following lemma provides an a priori estimate for the solution of (4.8).

Lemma 4.1. Let $(\phi, \beta)$ satisfy (4.8). Then, for $\delta$ sufficiently small, we have

$$
\|\phi\|_{*} \leq C\|h\|_{*} .
$$


Proof: We prove it by contradiction. Suppose not. Then there exists a sequence $\delta_{k} \rightarrow 0$ and a sequence of functions $\phi_{k}$ satisfying (4.8) such that the following holds:

$$
\left\|\phi_{k}\right\|_{*}=1, \quad\left\|h_{k}\right\|_{*}=o(1), \quad<\phi_{k}, Z_{i, j}>=0, \quad i=1, \ldots, K, j=1, \ldots, N .
$$

To keep notation simple, we drop the dependence on $k$.

Multiplying (4.8) by $\frac{\partial w_{k}}{\partial Q_{k, l}}$ and integrating over $R^{N}$, we obtain, using (4.3), that

$$
\sum_{i, j} \beta_{i j}<Z_{i j}, \frac{\partial w_{k}}{\partial Q_{k, l}}>=-<h, \frac{\partial w_{k}}{\partial Q_{k, l}}>+o(1)=O\left(\|h\|_{*}\right)+o(1) .
$$

Hence we obtain that

$$
|\beta|=O\left(\|h\|_{*}\right)+o(1)=o(1), \quad\left\|h+\sum_{i, j} \beta_{i j} Z_{i j}\right\|_{*}=o(1) .
$$

Therefore we have

$$
\left\|\Delta \phi-a \phi+g^{\prime}\left(w_{\delta, \mathbf{Q}}\right) \phi\right\|_{*}=o(1) .
$$

Since

$$
\left\|\left(g^{\prime}\left(w_{\delta, \mathbf{Q}}\right)-\sum_{j=1}^{K} g^{\prime}\left(w_{\delta, j}\right)\right) \phi\right\|_{*}=o(1)
$$

(4.12) is equivalent to

$$
\left\|\Delta \phi-a \phi+\sum_{j=1}^{K} g^{\prime}\left(w_{\delta, j}\right) \phi\right\|_{*}=o(1) .
$$

Fix an $R>0$. We claim that $\|\phi\|_{L^{\infty}\left(\cup_{j=1}^{K} B_{R}\left(Q_{j}\right)\right)}=o(1)$. In fact, suppose not. Then we may assume that $\|\phi\|_{L^{\infty}\left(B_{R}\left(Q_{1}\right)\right)} \geq c_{0}>0$. Thus as $\delta \rightarrow 0$, we have $\phi\left(y-Q_{1}\right) \rightarrow \phi_{0}$ in $C_{l o c}^{2}\left(R^{N}\right)$, where $\phi_{0}$ satisfies

$$
\Delta \phi_{0}-a \phi_{0}+g^{\prime}(w) \phi_{0}=0, \quad\left|\phi_{0}(y)\right| \leq C e^{-\mu|y|} .
$$

By Lemma 6.4 of [33], $\phi_{0}=\sum_{j=1}^{N} a_{j} \frac{\partial w}{\partial y_{j}}$. But $\int_{R^{N}} \phi_{0} g^{\prime}(w) \frac{\partial w}{\partial y_{j}}=0$ for $j=1, \ldots, N$. So $a_{j}=0, j=1, \ldots, N$. A contradiction.

Since $\|\phi\|_{L^{\infty}\left(\cup_{j=1}^{K} B_{R}\left(Q_{j}\right)\right)}=o(1)$, we obtain

$$
\left\|\sum_{j=1}^{K} g^{\prime}\left(w_{\delta, j}\right) \phi\right\|_{*}=o(1)
$$

and

$$
\|\Delta \phi-a \phi\|_{*}=o(1)
$$

By standard regularity theory (see Section 6 of [33]), $\|\phi\|_{*}=o(1)$. A contradiction.

Next we consider the existence problem for (4.8). 
Lemma 4.2. For $0<\delta<\delta_{0}$, given any $h \in L_{*}^{\infty}\left(R^{N}\right)$, there exists a unique pair $(\phi, c)$ such that the following hold:

$$
\begin{gathered}
L_{\delta}[\phi]=h+\sum_{i, j} \beta_{i, j} Z_{i, j}, \\
\phi \in H_{*}^{2}\left(R^{N}\right), \quad<\phi, Z_{i, j}>=0 .
\end{gathered}
$$

Moreover, we have

$$
\|\phi\|_{*} \leq C\|h\|_{*} .
$$

Proof: The existence follows from Fredholm's alternative. To this end, we set

$$
\mathcal{H}=\left\{u \in H_{*}^{1}\left(R^{N}\right) \mid<u, Z_{i, j}>=0, i=1, \ldots, K, j=1, \ldots, N\right\} .
$$

Observe that $\phi$ solves (4.17) and (4.18) if and only if $\phi \in H^{1}\left(R^{N}\right)$ satisfies

$$
\begin{gathered}
\int_{R^{N}}(\nabla \phi \nabla \psi+a \phi \psi)-<\left(g^{\prime}\left(w_{\delta, \mathbf{Q}}\right) \phi+\delta T[\phi], \psi>\right. \\
=<h, \psi>, \forall \psi \in H^{1}\left(R^{N}\right)
\end{gathered}
$$

This equation can be rewritten in the following form

$$
\phi+\mathcal{S}(\phi)=\bar{h}
$$

where $\mathcal{S}$ is a linear compact operator form $\mathcal{H}$ to $\mathcal{H}, \bar{h} \in \mathcal{H}$ and $\phi \in \mathcal{H}$.

Using Fredholm's alternative, to show equation that (4.20) has a unique solution for each $\bar{h}$, it is enough to show that the equation has a unique solution for $\bar{h}=0$. To this end, we assume the contrary. That is, there exists $(\phi, \beta) \in H_{*}^{2}\left(R^{N}\right)$ such that

$$
\begin{gathered}
L_{\delta}[\phi]=\sum_{i, j} \beta_{i j} Z_{i, j}, \\
<\phi, Z_{i, j}>=0, \quad i=1, \ldots, K, j=1, \ldots, N .
\end{gathered}
$$

From (4.21), it is easy to see that $\|\phi\|_{*}<+\infty$. So without loss of generality, we may assume that $\|\phi\|_{*}=1$. But then this contradicts (4.9).

Finally, we solve (4.6) for $(\phi, \beta)$. The following is the main result of this section.

Lemma 4.3. For $\mathbf{Q}=\left(Q_{1}, \ldots, Q_{K}\right) \in \bar{\Gamma}$ and $0<\delta<$ delta $_{0}$, there exists a unique pair $\left(\phi_{\delta, \mathbf{Q}}, \beta_{\delta}(\mathbf{Q})\right)$ solving (4.6). Furthermore, $\left(\phi_{\delta, \mathbf{Q}}, \beta_{\delta}(\mathbf{Q})\right)$ is continuous in $\mathbf{Q}$ and we have the following estimate

$$
\left\|\phi_{\delta, \mathbf{Q}}\right\|_{*} \leq \epsilon^{1-3 \mu} .
$$

Proof: We write (4.6) in the following form:

$$
L_{\delta}[\phi]=-S_{\delta}\left[w_{\delta, \mathbf{Q}}\right]-N_{\delta}[\phi]+\sum_{i, j} \beta_{i j} Z_{i, j}
$$


and use the contraction mapping theorem. Here $N_{\delta}[\phi]$ is given by

$$
N_{\delta}[\phi]=g\left(w_{\delta, \mathbf{Q}}+\phi\right)-g\left(w_{\delta, \mathbf{Q}}\right)-g^{\prime}\left(w_{\delta, \mathbf{Q}}\right) \phi .
$$

It is easy to see that

$$
\left\|N_{\delta}[\phi]\right\|_{*} \leq C\|\phi\|_{*}^{2} .
$$

Set $\mathcal{B}=\left\{\|\phi\|_{*}<\epsilon^{1-3 \mu}\right\} \subset L_{*}^{\infty}\left(R^{N}\right)$. We fix $\phi \in \mathcal{B}$ and consider the map $\mathcal{A}_{\delta}$ to be the unique solution given by Lemma 4.2 with $h=-S_{\delta}\left[w_{\delta, \mathbf{Q}}\right]-N_{\delta}[\phi]$. Then by Lemma 3.4 and by Lemma 4.2 , we have

$$
\left\|\mathcal{A}_{\delta}[\phi]\right\|_{*} \leq C\left\|-S_{\delta}\left[w_{\delta, \mathbf{Q}}\right]-N_{\delta}[\phi]\right\|_{*} \leq C \epsilon^{1-2 \mu}
$$

and hence $\mathcal{A}_{\delta}[\phi] \in \mathcal{B}$. Moreover, we also have that

$$
\left\|\mathcal{A}_{\delta}\left[\phi_{1}\right]-\mathcal{A}_{\delta}\left[\phi_{2}\right]\right\|_{*} \leq C\left\|N_{\delta}\left[\phi_{1}\right]-N_{\delta}\left[\phi_{2}\right]\right\|_{*} \leq \epsilon^{1-3 \mu}\left\|\phi_{1}-\phi_{2}\right\|_{*} .
$$

(4.27) and (4.28) show that the map $\mathcal{A}_{\delta}$ is a contraction map from $\mathcal{B}$ to $\mathcal{B}$. By the contraction mapping theorem, (4.24) has a unique solution $\phi \in \mathcal{B}$, called $\phi_{\delta, \mathbf{Q}}$.

The continuity of $\left(\phi_{\delta, \mathbf{Q}}, \beta_{\delta}(\mathbf{Q})\right)$ now follows from the continuity of $w_{\delta, \mathbf{Q}}$ and the uniqueness of $\left(\phi_{\delta, \mathbf{Q}}, \beta_{\delta}(\mathbf{Q})\right)$.

The last lemma shows the $C^{1}$-smoothness of $\phi_{\delta, \mathbf{Q}}$.

Lemma 4.4. For $0<\delta<\delta_{0}$, the map $\mathbf{Q}: \bar{\Gamma} \rightarrow \phi_{\delta, \mathbf{Q}}$ is actually $C^{1}$.

\section{Proof:}

Consider the map $H: \bar{\Gamma} \times H_{*}^{1}\left(R^{N}\right) \times R^{N K} \rightarrow H_{*}^{1}\left(R^{N}\right) \times R^{N K}$ defined by

$$
H(\mathbf{Q}, \phi, \beta)=\left(\begin{array}{c}
(\Delta-a)^{-1}\left(S_{\delta}\left[w_{\delta, \mathbf{Q}}+\phi\right]\right)+\sum_{i, j} \beta_{i j} \frac{\partial w_{i}}{\partial Q_{i, j}} \\
\left(\phi, \frac{\partial w_{i}}{\partial Q_{i, j}}\right)
\end{array}\right)
$$

which is of class $C^{1}$.

Equations (4.6) are equivalent to $H(\mathbf{Q}, \phi, \beta)=0$. We know that, given $\mathbf{Q} \in \bar{\Gamma}$, there is a unique local solution $\left(\phi_{\delta, \mathbf{Q}}, \beta_{\delta}(\mathbf{Q})\right)$ given by Lemma 4.3. We prove that the linear operator

$$
\left.\frac{\partial H(\mathbf{Q}, \phi, \beta)}{\partial(\phi, \beta)}\right|_{\left(\mathbf{Q}, \phi_{\delta, \mathbf{Q}}, \beta_{\delta}(\mathbf{Q})\right)}: H_{*}^{1}\left(R^{N}\right) \times R^{N K} \rightarrow H_{*}^{1}\left(R^{K}\right) \times R^{N K}
$$

is invertible for all $\mathbf{Q} \in \bar{\Gamma}$ and $\delta$ small. Then the $C^{1}$-regularity of $\mathbf{Q} \mapsto \phi_{\delta, \mathbf{Q}}$ follows from the Implicit Function Theorem. Indeed, we have

$$
\left.\frac{\partial H(\mathbf{Q}, \phi, \beta)}{\partial(\phi, \beta)}\right|_{\left(\mathbf{Q}, \phi_{\delta, \mathbf{Q}}, \beta_{\delta}(\mathbf{Q})\right)}[\hat{\phi}, \hat{\beta}]=\left(\begin{array}{c}
(\Delta-a)^{-1}\left(S_{\delta}^{\prime}\left[w_{\delta, \mathbf{Q}}+\phi_{\delta, \mathbf{Q}}\right](\hat{\phi})\right)+\sum_{i, j} \hat{\beta}_{i j} \frac{\partial w_{i}}{\partial Q_{i, j}} \\
\left(\hat{\phi}, \frac{\partial w_{i}}{\partial Q_{i, j}}\right)
\end{array}\right) .
$$

Since $\left\|\phi_{\delta, \mathbf{Q}}\right\|_{*}$ is small, the same proof as for Lemma 4.1 shows that $\left.\frac{\partial H(\mathbf{Q}, \phi, \beta)}{\partial(\phi, \beta)}\right|_{\left(\mathbf{Q}, \phi_{\delta, \mathbf{Q}}, \beta_{\delta}(\mathbf{Q})\right)}$ is invertible for $\delta$ small.

This concludes the proof of Lemma 4.4. 


\section{REDUCED ENERGY FUNCTIONAL}

In this section we expand the quantity

$$
M_{\delta}(\mathbf{Q}):=E_{\delta}\left[w_{\delta, \mathbf{Q}}+\phi_{\delta, \mathbf{Q}}\right]-K E\left[w_{\delta}\right]: \bar{\Gamma} \rightarrow R
$$

in $\delta$ and $\mathbf{Q}$, where $\phi_{\delta, \mathbf{Q}}$ is given by Lemma 4.3 .

We recall the condition (1.9) on the smallness of $\mu$ which will be used in the proof of Lemma 5.1.

We prove the following result by using Lemma 3.3 and estimating the error caused by adding $\phi_{\delta, \mathbf{Q}}$.

Lemma 5.1. Let $\phi_{\delta, \mathbf{Q}}$ be defined by Lemma 4.3. Then for any $\mathbf{Q}=\left(Q_{1}, \ldots, Q_{K}\right) \in \bar{\Gamma}$ and $0<\delta<\delta_{0}$ we have

$$
M_{\delta}(\mathbf{Q})=\delta c_{1} \sum_{i<j} \gamma\left(\left|Q_{i}-Q_{j}\right|\right)-\sum_{i<j} \alpha\left(Q_{i}-Q_{j}\right)+O\left(\epsilon^{1+4 \mu}\right),
$$

where $c_{1}>0$ is a positive constant and the functions $\alpha, \gamma$ are defined in (3.15), (3.16).

Proof. In fact, for any $\mathbf{Q} \in \bar{\Gamma}$, we have

$$
E_{\delta}\left(w_{\delta, \mathbf{Q}}+\phi_{\delta, \mathbf{Q}}\right)=E_{\delta}\left(w_{\delta, \mathbf{Q}}\right)+J_{\delta}\left(\phi_{\delta, \mathbf{Q}}\right)+O\left(\left\|\phi_{\delta, \mathbf{Q}}\right\|_{*}^{2}\right)
$$

where

We compute

$$
J_{\delta}\left(\phi_{\delta, \mathbf{Q}}\right)=\int_{R^{N}} S_{\delta}\left(w_{\mathbf{Q}}\right) \phi_{\delta, \mathbf{Q}} d y
$$

$$
\begin{aligned}
\left|J_{\delta}\left(\phi_{\delta, \mathbf{Q}}\right)\right|= & \left|\int_{R^{N}}\left(g\left(\sum_{i=1}^{K} w_{\delta, i}\right)-\sum_{i=1}^{K} g\left(w_{\delta, i}\right)\right) \phi_{\delta, \mathbf{Q}} d x\right| \\
& \leq C \epsilon^{1-2 \mu} \epsilon^{1-3 \mu} \leq C \epsilon^{1+4 \mu}
\end{aligned}
$$

by Lemma 3.2 and Lemma 4.3 .

The proof of Lemma 5.1 is completed.

The second and the last lemma in this section concerns the relation between the critical points of $M_{\delta}(\mathbf{Q})$ and those of the energy function $E_{\delta}[u]$.

Lemma 5.2. Suppose $\mathbf{Q}^{\delta} \in$ int $(\Gamma)$ is a critical point of $M_{\delta}(\mathbf{Q})$ and $0<\delta<\delta_{0}$. Then the corresponding function $u_{\delta}=w_{\delta, \mathbf{Q}^{\delta}}+\phi_{\delta, \mathbf{Q}^{\delta}}$ is also a critical point of $E_{\delta}[u]: H^{1}\left(R^{N}\right) \rightarrow R$ and hence a solution of (1.2).

\section{Proof:}

By Lemma 4.3 and Lemma 4.4, there exists a $\delta_{0}>0$ such that for $0<\delta<\delta_{0}$ we have a $C^{1}$ map which, to any $\mathbf{Q} \in \bar{\Gamma}$, associates $\phi_{\delta, \mathbf{Q}}$ such that

$$
S_{\delta}\left(w_{\delta, \mathbf{Q}}+\phi_{\delta, \mathbf{Q}}\right)=\sum_{i, j} \beta_{i j} Z_{i, j}
$$

for some constants $\beta_{i j} \in R^{N K}$.

Let $\mathbf{Q}^{\delta} \in \Gamma$ be a critical point of $M_{\delta}(\mathbf{Q})$. Let $u_{\delta}=w_{\delta, \mathbf{Q}^{\delta}}+\phi_{\delta, \mathbf{Q}^{\delta}}$. Then we have

$$
\left.\frac{\partial}{\partial Q_{i, j}}\right|_{\mathbf{Q}=\mathbf{Q}^{\delta}} M_{\delta}\left(\mathbf{Q}^{\delta}\right)=0, \quad i=1, \ldots, K, \quad j=1, \ldots, N .
$$


Hence we have

which is equivalent to

$$
\begin{gathered}
\int_{R^{N}}\left[\left.\nabla u_{\delta} \nabla \frac{\partial\left(w_{\delta, \mathbf{Q}}+\phi_{\delta, \mathbf{Q}}\right)}{\partial Q_{i, j}}\right|_{\mathbf{Q}=\mathbf{Q}^{\delta}}\right. \\
\left.+\left.\left(-a u_{\delta}+g\left(u_{\delta}\right)-\delta T\left[u_{\delta}\right]\right) \frac{\partial\left(w_{\delta, \mathbf{Q}}+\phi_{\delta, \mathbf{Q}}\right)}{\partial Q_{k, l}}\right|_{\mathbf{Q}=\mathbf{Q}^{\delta}}\right]=0
\end{gathered}
$$

Thus we have from $(5.4)$$$
\left.\int_{R^{N}} S_{\delta}\left(u_{\delta}\right) \frac{\partial\left(w_{\delta, \mathbf{Q}}+\phi_{\delta, \mathbf{Q}}\right)}{\partial Q_{k, l}}\right|_{\mathbf{Q}=\mathbf{Q}^{\delta}}=0
$$

$$
\left.\sum_{i, j} \beta_{i j} \int_{R^{N}} Z_{i, j}\left(\frac{\partial\left(w_{\delta, \mathbf{Q}}+\phi_{\delta, \mathbf{Q}}\right)}{\partial Q_{k, l}}\right)\right|_{\mathbf{Q}=\mathbf{Q}^{\delta}}=0
$$

Since $<Z_{i, j}, \phi_{\delta, \mathbf{Q}}>=0$, we have for $\mathbf{Q}=\mathbf{Q}^{\delta}$ that

$$
\int_{R^{N}} Z_{i, j} \frac{\partial \phi_{\delta, \mathbf{Q}}}{\partial Q_{k, l}}=-\int_{R^{N}} \phi_{\delta, \mathbf{Q}} \frac{\partial Z_{i, j}}{\partial Q_{k, l}}=O\left(\epsilon^{1-3 \mu}\right)
$$

as $\left\|\phi_{\delta, \mathbf{Q}}\right\|_{*}=O\left(\epsilon^{1-3 \mu}\right)$.

Note that

where

$$
\int_{R^{N}} Z_{i, j} \frac{\partial w_{\delta, \mathbf{Q}}}{\partial Q_{k, l}}=\delta_{i k} \delta_{j l} A_{0}(1+o(1))
$$

$$
A_{0}=\int_{R^{N}} g^{\prime}(w)\left(\frac{\partial w}{\partial y_{1}}\right)^{2}=\int_{R^{N}}\left[\left|\nabla\left(\frac{\partial w}{\partial y_{1}}\right)\right|^{2}+a\left(\frac{\partial w}{\partial y_{1}}\right)^{2}\right]>0
$$

Thus (5.5) becomes a system of homogeneous equations for $\beta_{i j}$ and the matrix of the system is nonsingular since it is dominated by its diagonal. So $\beta_{i j}=0, i=1, \ldots, K, j=1, \ldots N$.

Hence $u_{\delta}=w_{\delta, \mathbf{Q}^{\delta}}+\phi_{\delta, \mathbf{Q}^{\delta}}$ is a solution of (1.2).

\section{The Reduced Problem: Proof of Theorem 1.1}

In this section, we study a maximization problem.

Fix $\mathbf{Q} \in \bar{\Gamma}$. Let $\Phi_{\delta, \mathbf{Q}}$ be the solution given by Lemma 4.3. We shall prove

Proposition 6.1. For $0<\delta<\delta_{0}$, the following maximization problem

$$
\max \left\{M_{\delta}(\mathbf{Q}) \mid \mathbf{Q} \in \bar{\Gamma}\right\}
$$

has a solution $\mathbf{Q}^{\delta}$ which belongs to the interior of $\Gamma$. Furthermore we have

$$
\lim _{\delta \rightarrow 0} \frac{l^{\delta}}{\log \frac{1}{\delta}}=\frac{1}{\sqrt{a}-\beta} \text {, where } l^{\delta}=\min _{i \neq j}\left|Q_{i}^{\delta}-Q_{j}^{\delta}\right|
$$

and, possibly after renumbering indices, for some subsequence we have $\frac{\mathbf{Q}^{\delta}}{l^{\delta}} \rightarrow \mathbf{Q}^{0}$ with

$$
R\left[Q_{1}^{0}, \ldots, Q_{K}^{0}\right]=m(N, K)
$$

where the number $m(N, K)$ is defined in (2.3). 
Before we prove the above proposition, we present one lemma on a finite dimensional problem.

Lemma 6.2. Consider the function

$$
h(\rho):=c_{1} \delta \gamma(\rho)-\alpha(\rho), \quad \rho \geq \frac{1-\mu}{\sqrt{a}-\beta} \log \frac{1}{\delta},
$$

where $c_{1}$ is a positive constant introduced in Lemma 3.3.

Then, for $0<\delta<\delta_{0}, h(\rho)$ has a unique maximum point $\rho_{\max }$. Moreover we have

$$
\rho_{\max }=\frac{1}{\sqrt{a}-\beta} \log \frac{1}{\delta}+O\left(\log \log \frac{1}{\delta}\right)
$$

and

$$
h\left(\rho_{\max }\right)=c_{3} \delta^{\frac{\sqrt{a}}{\sqrt{a}-\beta}}\left(\log \frac{1}{\delta}\right)^{-(N-1) / 2}(1+o(1))=c_{3} \epsilon\left(\log \frac{1}{\epsilon}\right)^{-(N-1) / 2}(1+o(1))
$$

for some positive constant $c_{3}>0$.

Proof: This is a calculus problem since for $\rho$ large, since by Lemma 3.2,

$$
\begin{gathered}
\alpha(\rho) \sim w(\rho)=A_{N} \rho^{-\frac{N-1}{2}} e^{-\sqrt{a} \rho}\left(1+O\left(\frac{1}{\rho}\right)\right), \\
\gamma(\rho) \sim \Psi(\rho)=B_{N} \rho^{-\frac{N-1}{2}} e^{-\beta \rho}\left(1+O\left(\frac{1}{\rho}\right)\right) .
\end{gathered}
$$

Moreover these estimates hold in the $C^{2}$ sense.

Differentiation of the function $h(\rho)$ gives an equation for the critical point of $h(\rho)$ :

$$
-\beta B_{N}\left(1+O\left(\frac{1}{\rho}\right)\right) \rho^{-\frac{N-1}{2}} c_{2} \delta e^{-\beta \rho}+A_{N}\left(1+O\left(\frac{1}{\rho}\right)\right) \sqrt{a} \rho^{-\frac{N-1}{2}} e^{-\sqrt{a} \rho}=0 .
$$

Taking the logarithm, (6.5) and (6.6) follow by an elementary computation.

\section{Proof of Proposition 6.1:}

Since the set $\Gamma$ is compact, the function $M_{\delta}(\mathbf{Q})$ has a maximum point $\mathbf{Q}^{\delta} \in \bar{\Gamma}$. We first show that $\mathbf{Q}^{\delta}$ must lie in the interior of $\Gamma$.

We first obtain a lower bound for $M_{\delta}\left(\mathbf{Q}^{\delta}\right)$. Let $\mathbf{Q}^{0}=\left(Q_{1}^{0}, \ldots, Q_{K}^{0}\right)$ be an optimal configuration given by Lemma 2.1. We choose $\mathbf{Q}=\rho_{\max } \mathbf{Q}^{0}$, where $\rho_{\max }$ is given by Lemma 6.2. It is easy to see that this choice of $\mathbf{Q}$ belongs to $\Gamma$. Then we have

$$
\begin{gathered}
M_{\delta}\left(\mathbf{Q}^{\delta}\right) \geq \sum_{i \neq j} h\left(\rho_{\max }\left|Q_{i}^{0}-Q_{j}^{0}\right|\right)+O\left(\epsilon^{1+4 \mu}\right) \\
\geq m(N, K) h\left(\rho_{\max }\right)(1+o(1)) \geq c_{3} m(N, K) \epsilon\left(\log \frac{1}{\epsilon}\right)^{-(N-1) / 2}(1+o(1))
\end{gathered}
$$

by Lemma 6.2 .

Let $l^{\delta}=\min _{i \neq j}\left|Q_{i}^{\delta}-Q_{j}^{\delta}\right|$. Then $l^{\delta}>(1-\mu) \frac{1}{\sqrt{a}-\beta} \log \frac{1}{\delta}$. Without loss of generality, we may assume that $l^{\delta}=\left|Q_{1}^{\delta}-Q_{2}^{\delta}\right|$. 
In fact, suppose not. Then we have

$$
M_{\delta}\left(\mathbf{Q}^{\delta}\right) \leq\left(\sum_{i \neq j,(i, j) \neq(1,2)}\left[h\left(\rho_{\max }\right)\right]\right)+h\left(l^{\delta}\right)+O\left(\epsilon^{1+4 \mu}\right) \leq-\delta^{\left(1-\frac{\mu}{2}\right) \frac{\sqrt{a}}{\sqrt{a}-\beta}}(1+o(1)) \leq-\epsilon^{1-\frac{\mu}{2}}(1+o(1))
$$

which contradicts (6.7).

Consider the rescaled vertex $\hat{Q}_{i}^{\delta}=\frac{1}{l^{\delta}} Q_{i}^{\delta}$. Let us assume that $\lim _{\delta \rightarrow 0}\left\{\hat{Q}_{i}^{\delta}, i=1, \ldots, K\right\}=\left\{\hat{Q}_{i_{l}}^{0}, l=1, \ldots, k\right\}$. Note that $k \geq 2$.

Suppose first that $k<K$. We will show that this is impossible. We get

$$
M_{\delta}\left(\mathbf{Q}^{\delta}\right) \leq h\left(l^{\delta}\right) R\left[\hat{Q}_{1}^{0} \ldots, \hat{Q}_{K}^{0}\right]+o\left(h\left(l^{\delta}\right)\right) \leq h\left(l^{\delta}\right) m(N, k)+o\left(h\left(l^{\delta}\right)\right) .
$$

Comparing (6.7) and (6.9), we conclude that

$$
h\left(\rho_{\max }\right) m(N, K) \leq h\left(l^{\delta}\right) m(N, k)
$$

which is impossible since $h\left(l^{\delta}\right) \leq h\left(\rho_{\max }\right)$ and by $(2.4)$ we know that $m(N, K)>m(N, k)$.

Because of the connectedness of the graph of shortest distances (see Lemma 2.1), if $\left|Q_{i}-Q_{j}\right|=\left(\log \frac{1}{\delta}\right)^{2}$ then $k<K$. This together with (6.8) shows that $\mathbf{Q}^{\delta}$ must lie in the interior of $\Gamma$.

We now show (6.2) and (6.3). If $k=K$, then $l^{\delta} \leq\left|Q_{i}^{\delta}-Q_{j}^{\delta}\right| \leq C \log \frac{1}{\delta}$ and hence $\mathbf{Q}^{\delta}$ belongs to the interior of $\Gamma$. In this case, we then have

$$
M_{\delta}\left(\mathbf{Q}^{\delta}\right) \leq h\left(l^{\delta}\right) m(N, K)+o\left(h\left(l^{\delta}\right)\right) .
$$

From (6.7) and (6.11), we deduce that $\limsup _{\delta \rightarrow 0} \frac{l^{\delta}}{\rho_{\max }} \leq 1$ and

$$
R\left[\hat{Q}_{1}^{0}, \ldots, \hat{Q}_{K}^{0}\right]=m(N, K) .
$$

Now (6.3) is proved. We also observe that $\lim _{\delta \rightarrow 0} \frac{l^{\delta}}{\rho_{\max }}=1$ as otherwise $\liminf _{\delta \rightarrow 0} \frac{l^{\delta}}{\rho_{\max }}<1$ and then a similar argument as the one leading to (6.8) gives a contradiction. This implies (6.2).

\section{Completion of the Proof of Theorem 1.1:}

Theorem 1.1 is proved by combining Proposition 6.1 and Lemma 5.2.

\section{Proof of Theorem 1.2}

In this section we are going to construct solutions which have certain symmetries. Therefore we proceed in weighted Sobolev spaces of symmetric functions.

We require symmetry by rotation of $2 \pi / K$ and by reflection. Therefore, using complex notation $z=y_{1}+$ $\sqrt{-1} y_{2}$, we introduce the spaces

$$
\begin{gathered}
X=\left\{u\left(y_{1}, y_{2}, \ldots, y_{N}\right) \mid u\left(y_{1}, y_{2}, y^{\prime}\right)=u\left(z,\left|y^{\prime}\right|\right), \quad u\left(z e^{2 \pi \sqrt{-1} / K},\left|y^{\prime}\right|\right)=u\left(z,\left|y^{\prime}\right|\right), \quad u\left(\bar{z},\left|y^{\prime}\right|\right)=u\left(z,\left|y^{\prime}\right|\right)\right\} \\
H_{*, s}^{2}\left(R^{N}\right)=H_{*}^{2}\left(R^{N}\right) \cap X, L_{*, s}^{\infty}\left(R^{N}\right)=L_{*}^{\infty}\left(R^{N}\right) \cap X .
\end{gathered}
$$

Now we can construct a solution in these symmetric spaces following the Liapunov-Schmidt reduction in Section 5. 
Then the functional $M_{\delta}(\mathbf{Q})=E_{\delta}\left(w_{\delta, \mathbf{Q}}+\Phi_{\delta, \mathbf{Q}}\right)$ defined in (5.1) also has these symmetries. Moreover, we may assume that all the vertices lie in the two-dimensional plane $\left\{\left(y_{1}, y_{2}, y^{\prime}\right) \mid y^{\prime}=0\right\}$. That is $Q_{i}=\left(Q_{i, 1}, Q_{i, 2}, 0, \ldots, 0\right)=$ $\left(\tilde{Q}_{i}, 0^{\prime}\right)$. To simplify notation, we denote $\tilde{Q}=\left(Q_{i, 1}, Q_{i, 2}\right)$ as $\mathbf{Q}$. Then we have

$$
\begin{gathered}
M_{\delta}\left(\mathbf{Q} e^{2 \pi \sqrt{-1} / K}\right)=M_{\delta}(\mathbf{Q}), \\
M_{\delta}(\overline{\mathbf{Q}})=M_{\delta}(\mathbf{Q}),
\end{gathered}
$$

where multiplication and complex conjugation are applied to every component of $\mathbf{Q}$ separately.

Let the points $\left(\mathbf{Q}(\rho)=Q_{1}(\rho), \ldots, Q_{K}(\rho)\right)$ form a regular polygon with side length $\rho$. It is easy to compute that

$$
M_{\delta}(\mathbf{Q}(\rho))=K \sum_{j=2}^{K / 2} h\left(\left|Q_{1}(\rho)-Q_{j}(\rho)\right|\right)+O\left(\epsilon^{1+4 \mu}\right) .
$$

Set

$$
\begin{gathered}
\Gamma_{1}:=\left\{\mathbf{Q}(\rho)=\left(Q_{1}(\rho), \ldots, Q_{K}(\rho)\right) \mid Q_{i}(\rho)=\frac{\rho}{2 \sin (\pi / K)} e^{2 \pi i \sqrt{-1} / K}, \quad i=1,2, \ldots, K,\right. \\
\left.(1-\mu) \frac{1}{\sqrt{a}-\beta} \log \frac{1}{\delta} \leq \rho \leq\left(\log \frac{1}{\delta}\right)^{2}\right\} .
\end{gathered}
$$

We then maximize $M_{\delta}(\mathbf{Q}(\rho))$ over $\Gamma_{1}$. On $\partial \Gamma_{1}$ we have either $\rho=(1-\mu) \frac{1}{\sqrt{a}-\beta} \log \frac{1}{\delta}$ or $\rho=\left(\log \frac{1}{\delta}\right)^{2}$. Computations as in Section 6 show that in the first case $M_{\delta}(\mathbf{Q}(\rho))<-\epsilon^{1-\frac{\mu}{2}}$ and in the second case

$$
M_{\delta}(\mathbf{Q}(\rho)) \leq C \frac{\delta}{\left(\log \frac{1}{\delta}\right)^{N-1}}
$$

On the other hand, for the maximum point $\mathbf{Q}\left(\rho_{m}\right)$ we estimate by the same proof as in Proposition 6.1 that

$$
M_{\delta}\left(\mathbf{Q}\left(\rho_{m}\right)\right)=K h\left(\rho_{\max }\right)(1+o(1))
$$

since for the polygon with $K$ vertices the minimal distance is attained exactly $K$ times.

Therefore the maximum of $M_{\delta}(\mathbf{Q}(\rho))$ can not be obtained for $\mathbf{Q}(\rho) \in \partial \Gamma_{1}$. Therefore it must be obtained in the interior. This gives rise to a critical point of $M_{\delta}(\mathbf{Q})$. By Lemma 5.2, we obtain a solution to (1.2) with $K$-vertices on a regular polygon.

To construct two concentric polygons we set

$$
\begin{gathered}
\Gamma_{2}:=\left\{\begin{array}{c}
\mathbf{Q}\left(\rho_{1}, \rho_{2}\right)=\left(Q_{1}\left(\rho_{1}\right), \ldots, Q_{K / 2}\left(\rho_{1}\right), Q_{K / 2+1}\left(\rho_{2}\right), \ldots, Q_{K}\left(\rho_{2}\right)\right) \mid \\
Q_{i}(\rho)=\frac{\rho_{1}}{2 \sin (2 \pi / K)} e^{4 \pi i \sqrt{-1} / K}, i=1, \ldots, K / 2, \\
Q_{i}(\rho)=\frac{\rho_{2}}{2 \sin (2 \pi / K)} e^{4 \pi i \sqrt{-1} / K}, i=K / 2+1, \ldots, K, \\
(1-\mu) \frac{1}{\sqrt{a}-\beta} \log \frac{1}{\delta} \leq \rho_{1} \leq\left(\log \frac{1}{\delta}\right)^{2}, \\
(1-\mu) \frac{1}{\sqrt{a}-\beta} \log \frac{1}{\delta} \leq \frac{\rho_{2}-\rho_{1}}{2 \sin (2 \pi / K)} \leq\left(\log \frac{1}{\delta}\right)^{2} .
\end{array} .\right.
\end{gathered}
$$


The reduced energy $M_{\delta}(\mathbf{Q})$ takes the following form

$$
\begin{gathered}
M_{\delta}(\mathbf{Q})=K \sum_{j=2}^{K / 2} h\left(\left|Q_{1}\left(\rho_{1}\right)-Q_{j}\left(\rho_{1}\right)\right|\right) \\
+K \sum_{j=K / 2+1}^{K} h\left(\left|Q_{1}\left(\rho_{1}\right)-Q_{j}\left(\rho_{2}\right)\right|\right)+K \sum_{j=1}^{K / 2} h\left(\left|Q_{K / 2+1}\left(\rho_{2}\right)-Q_{j}\left(\rho_{1}\right)\right|\right) \\
+K \sum_{j=K / 2+2}^{K} h\left(\left|Q_{K / 2+1}\left(\rho_{2}\right)-Q_{j}\left(\rho_{2}\right)\right|\right)+O\left(\epsilon^{1+4 \mu}\right) .
\end{gathered}
$$

Observe that if $\rho_{2}-\rho_{1}=(1-\mu) \frac{1}{\sqrt{a}-\beta} \log \frac{1}{\delta}$, then

$$
h\left(\left|Q_{\frac{K}{2}+1}\left(\rho_{2}\right)-Q_{j}\left(\rho_{1}\right)\right|\right) \leq-\epsilon^{1-\mu / 2}
$$

The rest of the argument is similar to that for the single polygon.

The proof of Theorem 1.2 is finished.

\section{REFERENCES}

[1] Alikakos, N., Fusco, G., and Kowalczyk, M., Finite dimensional dynamics and interfaces intersecting the boundary I, Indiana Univ. Math. J. 45 (1996), 1119-1155.

[2] N. Alikakos and M. Kowalczyk, Critical points of a singular perturbation problem via reduced energy and local linking, J. Differential Equations 159 (1999), 403-426.

[3] P. Bates, E.N. Dancer and J. Shi, Multi-spike stationary solutions of the Cahn-Hilliard equation in higher-dimension and instability, Adv. Differential Equations 4 (1999), 1-69.

[4] P. Bates and G. Fusco, Equilibria with many nuclei for the Cahn-Hilliard equation, J. Differential Equations. 4 (1999), 1-69.

[5] P. Bates and J. Shi, Existence and instability of spike layer solutions to singular perturbation problems, J. Funct. Anal., 196 (2002), 211-264.

[6] C. Bender, Bestimmung der größten Zahl gleich großer Kugeln, welche sich auf eine Kugel von demselben Radius wie die übrigen auflegen lassen, Grunert Arch. 56 (1874), 302-313.

[7] X. Chen, M. del Pino and M. Kowalczyk, The Gierer and Meinhardt system: the breaking of homoclinics and multi-bump ground states, Commun. Contemp. Math. 3 (2001), 419-439.

[8] P. Clement and G. Sweers, Existence and multiplicity results for a semilinear eigenvalue problem, Ann. Scuola Norm. Sup. Pisa 14 (1987), 97-121.

[9] J.H. Conway and N.J.A. Sloan, Sphere packings, lattices and groups, Die Grundlehren der mathematischen Wissenschaften in Einzeldarstellungen 290, Springer, New York, Heidelberg, 3rd ed., 1999.

[10] H.S.M. Coxeter, An upper bound for the number of equal nonoverlapping spheres that can touch another of the same size, Convexity, Proc. Symp. Pure Math. 7 (1963), 53-71.

[11] E.N. Dancer, A note on the asymptotic uniqueness for some nonlinearities which change sign, Bull. Austral. Math. Soc. 61 (2000), 305-312.

[12] A. Doelman, T.J. Kaper, and H. van der Ploeg, Spatially periodic and aperiodic multi-pulse patterns in the one-dimensional Gierer-Meinhardt equation, Methods Appl. Anal., 8 (2001), 387-414.

[13] E.N. Dancer and S. Yan, Multipeak solutions for a singular perturbed Neumann problem, Pacific J. Math. 189 (1999), $241-262$.

[14] E.N. Dancer and S. Yan, Interior and boundary peak solutions for a mixed boundary value problem, Indiana Univ. Math. J. 48 (1999), 1177-1212.

[15] E.N. Dancer and S. Yan, A minimization problem associated with elliptic systems of FitzHugh-Nagumo type, Ann. Inst. H. Poincaré Anal. Non Linéaire, to appear.

[16] D.G. deFigueiredo and E. Mitidieri, A Maximum Principle for an elliptic system and applications to semilinear problems, SIAM J. Math. Anal. 17 (1986), 74-83.

[17] M. del Pino, M. Kowalczyk and J. Wei, Multi-bump ground states for the Gierer-Meinhardt system in $R^{2}$, Ann. Inst. H. Poincaré Anal. Non Linéaire 20 (2003), 53-85.

[18] L. Fejes-Toth, New results in the theory of packing and covering, in: Convexity and its Applications, eds. P.M. Gruber and J.M. Wills (Birkhäuser, Basel) pp. 318-359, 1983.

[19] R. FitzHugh, Impulse and physiological states in models of nerve membrans, Biophysics J. 1 (1961), 445-466. 
[20] Floer, A. and Weinstein, A., Nonspreading wave packets for the cubic Schrödinger equation with a bounded potential, J. Funct. Anal. 69 (1986), 397-408.

[21] C. Gui and J. Wei, Multiple interior peak solutions for some singular perturbation problems, J. Differential Equations. 158 (1999), 1-27.

[22] C. Gui and J. Wei, On multiple mixed interior and boundary peak solutions for some singularly perturbed Neumann problems, Canad. J. Math. 52 (2000), 522-538.

[23] C. Gui, J. Wei and M. Winter, Multiple boundary peak solutions for some singularly perturbed Neumann problems, Ann. Inst. H. Poincaré Anal. Non Linéaire 17 (2000), 47-82.

[24] P. Erdös and G. Purdy, Extremal problems in combinatorial geometry, in: Handbook of Combinatorics, eds. R. Graham, M. Grötschel and L. Lovász (Elsevier Science, B.V.), pp. 809-871, 1995.

[25] H. Harborth, Solution to problem 664a, Elemente Math. 29 (1974), 14-15.

[26] A.L. Hodgkin and A.F. Huxley, A quantitative description of membrane current and its application to conduction and excitation in nerve, J. Physiol. 117 (1952), 500-544.

[27] G.A. Klaasen and E. Mitidieri, Standing wave solutions for a system derived from the FitzHugh-Nagumo equations for nerve conduction, SIAM J. Math. Anal. 17 (1986), 74-83.

[28] G.A. Klaasen and E. Mitidieri, Standing wave solutions of a system of reaction-diffusion equations derived from the FitzHughNagumo equations, SIAM J. Appl. Math. 44 (1986), 74-83.

[29] M. Kowalczyk, Multiple spike layers in the shadow Gierer-Meinhardt system: existence of equilibria and approximate invariant manifold, Duke Math. J. 98(1999), 59-111.

[30] A.C. Lazer and P.J. McKenna, On steady state solutions of a system of reaction-diffusion equations from biology, Nonlinear Analysis 6 (1982), 523-530.

[31] J. Leech, The problem of the thirteen spheres, Math. Gaz. 40 (1956), 22-23.

[32] J.S. Nagumo, S. Arimoto, and S. Yoshizawa, An active pulse transmission line simulating nerve axon, Proc. IRE 50 (1962), 2061-2071.

[33] W.-M. Ni and J. Wei, On the location and profile of spike-layer solutions to singularly perturbed semilinear Dirichlet problems, Comm. Pure. Appl. Math. 48 (1995), 731-768.

[34] Y. Nishiura, Coexistence of infinitely many stable solutions to reaction-diffusion system in the singular limit, Dynamics Reported: Expositions in Dynamical Systems, Edited by C.R.K.T. Jones, U. Kirchgraber and H.O. Walther, Volume 3, SpringerVerlag, New York, 1994.

[35] Y. Nishiura and H. Fujii, Stability of singularly perturbed solutions to systems of reaction diffusion euations, SIAM J. Math. Anal. 18 (1987), 1726-1770.

[36] T. Ouyang and J. Shi, Exact multiplicity of positive solutions for a class of semilinear problems, J. Differential Equations 146 (1998), 121-156.

[37] Y. Oshita, On stable nonconstant stationary solutions and mesoscopic patterns for FitzHugh-Nagumo equations in higher dimensions, J. Differential Equations 188 (2003), 110-134.

[38] Y. Oshita and I. Ohnishi, Standing pulse solutions for the FitzHugh-Nagumo euations, Jap. J. Ind. Appl. Math. 20 (2003), $101-115$.

[39] G. Sweers and W.C. Troy, On the bifurcation curve for an elliptic system of FitzHugh-Nagumo type, Phys. D 177 (2003), 1-22.

[40] C. Reinecke and G. Sweers, Solutions with internal jump for an autonomous elliptic system of FitzHugh-Nagumo type, Math. Nachr. 254 (2003), 64-87.

[41] C. Reinecke and G. Sweers, Existence and uniqueness of solutions on bounded domains to a FitzHugh-Nagumo type elliptic system, Pacific J. Math. 197 (2001), 183-211.

[42] C. Reinecke and G. Sweers, A boundary layer solution to a semilinear elliptic system of FitzHugh-Nagumo type, C. R. Acad. Sci. Paris Ser. I Math. 329 (1999), 27-32.

[43] C.Reinecke and G. Sweers, Positive solution on $R^{N}$ to a system of elliptic equations of FitzHugh-Nagumo type, J. Differential Equations 153 (1999), 292-312.

[44] J. Wei and M. Winter, Stationary solutions for the Cahn-Hilliard equation, Ann. Inst. H. Poincaré Anal. Non Linéaire 15 (1998), 459-492.

[45] J. Wei and M. Winter, Multiple boundary spike solutions for a wide class of singular perturbation problems, J. London Math. Soc. 59 (1999), 585-606.

[46] J. Wei and M. Winter, Symmetric and asymmetric multiple clusters in a reaction-diffusion system, NoDEA Nonlinear Differential Equations Appl., to appear.

School of Information Systems, Computing and Mathematics, Brunel University, Uxbridge UB8 3PH, United Kingdom E-mail address: matthias.winter@brunel.ac.uk

Department of Mathematics, The Chinese University of Hong Kong, Shatin, Hong Kong

E-mail address: wei@math.cuhk.edu.hk 